\title{
Traffic Video Enhancement based Vehicle Correct Tracked Methodology
}

\author{
Mohamed Maher Ata \\ Faculty of Engineering, Tanta University, Egypt. \\ Email: mmaher844@yahoo.com \\ Mohamed El-Darieby \\ Faculty of Engineering, University of Regina, Canada. \\ Email: Mohamed.El-Darieby@uregina.ca \\ M.Abd Elnaby and Sameh A. Napoleon \\ Faculty of Engineering, Tanta University, Egypt. \\ Email: mnaby45@gmail.com, s.napoleon@f-eng.tanta. edu.eg
}

Received: 08 September 2017; Accepted: 22 September 2017; Published: 08 December 2017

\begin{abstract}
In this paper, an enhancement based traffic video has been proposed in the state of the art of computer vision. The main target is to develop a decision making criteria for removing the most probable video degradations. Such traffic video degradations would have an adverse impact on the transportation system. In order to establish the appropriate analysis, three types of video degradations have been added to the test video; salt and pepper noise, Gaussian noise, and speckle noise, we have simulated rainy, fog, and darkness conditions for the traffic video. First of all, back ground subtraction and Kalman filter techniques have been used for detecting and tracking vehicles respectively. By using such algorithms, it would be easily to estimate average number of assigned tracks which express the efficacy of correct detection and prediction of vehicles in each frame. Furthermore, video degradations would be applied in order to studying its effect on the average number of assigned tracks which would be deviated than noiseless video. Spatial filtering system has been applied to state the most suitable filter mask which satisfy the least deviation in the average number of assigned tracks. Experimental results show that median filter satisfies the least deviation in all cases of video degradations.
\end{abstract}

Index Terms - Intelligent Transportation System (ITS), traffic video analysis, assigned tracks, video degradation, filter mask.

\section{INTRODUCTION}

Traffic video analysis would be considered as one of the most challenging research point in this century. Such analysis has been targeting an intelligent transportation system (ITS) which serves both the traffic system infrastructure and drivers themselves [1]. Many smart techniques have been designed in order to detect and keep track moving vehicles from the first frame until reaching the last frame in the test video [2]. Some of these techniques would be software based systems or hardware based systems. Our proposed system was targeting to be a software based analysis according to means of computer vision techniques. In This paper, an extended study has been proposed in the state of the art of computer vision. The proposed Study aims to produce a decision tool in order to enhance traffic video with the presence of video degradations. In the beginning, we have applied a noiseless traffic video to both vehicle detection algorithms based foreground detection and vehicle tracking based Kalman filter. In addition, some sort of statistical information has been extracted to express number of correct detected and predicted vehicles (number of assigned tracks in each frame). By applying means of averaging, we can now calculate the average number of assigned tracks in the whole video in order to having a reference value that express the accuracy of detection and tracking of vehicles. The next stage has been utilized by applying means of video degradation; salt and pepper, Gaussian noise, and speckle noise. In each case, Kalman filter has been applied to measure average number of assigned tracks. In the last stage, some filter masks have been applied to the noisy video in order to estimate the best filtering algorithm for such traffic video by calculating the deviation between values of average number of assigned tracks in both noiseless video and cleaned video. The least deviation value would express the best filter mask. Figure 1 shows the block diagram of the proposed system. 


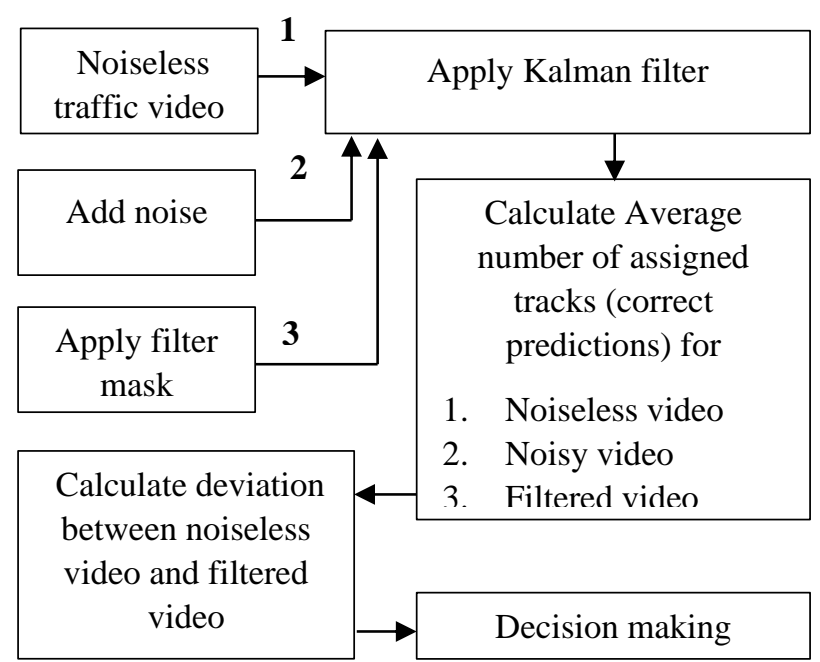

Fig.1. block diagram of the proposed system

\section{Techniques of Detecting and TRacking Vehicles}

Foreground detection based Gaussian mixture back ground subtraction technique is one of the most popular algorithms which used in the detection of multiple vehicles. In this technique, successive video frames are subsequent to multiple separately frames. In addition, each frame would be compared with other frames in order to detect moving vehicles. Gaussian mixture model relies on 2 main steps; initialize foreground detector, and detecting vehicles in an initial video frames [3]. In the first step, moving vehicles are segmented from the background [4,5]. For accepted results, the chosen values of the appropriate parameters must be estimated relatively to the total number of successive video frames of interest (about $10 \%$ of the total number of video frames) [6]. By applying some sort of training process, the foreground detector starts to output segmented vehicles. In the second step, vehicle detection would be concerned. After applying foreground detection, a blob analyzer algorithm would be applied in order to remove any undesired moving objects in the video [7]. The final phase in the detection procedure is to fill undetectable discontinuous regions with holes in vehicle window by means of dilation and erosion [8]. By completing the detection process, a tracking algorithm based on Kalman filter would be applied to ensure that each vehicle would be kept tracked $[9,10]$. To ensure the process of tracking vehicles regardless the change of position, speed and acceleration, we measure the distance of centroids of each vehicle blob that calculated in two consecutive frames $[11,12]$. If two centroids $(x 1, y 1)$ and $(x 2, y 2)$ of two vehicle blobs, then distance would be calculated as follow:

$$
d=\sqrt{\left[\left(y_{2}-y_{1}\right)^{2}+\left(x-x_{1}\right)^{2}\right]}
$$

\section{VIDEO DEGRADATION AND SUGGESTED FILTERING TECHNIQUES}

Video degradations represent the external disturbance which affect the successive video frames [13]. Salt \& pepper noise (shot noise or binary noise) can be caused by sharp and sudden disturbances in the video frames. Its effect represented by scattered dots. Gaussian noise (additive noise) can be caused by random fluctuations. It is a normally distributed white noise. Principal sources of Gaussian noise in digital images arise during acquisition e.g. sensor noise caused by poor illumination and/or high temperature, and/or transmission e.g. electronic circuit noise Speckle noise (multiplicative noise) can be caused by random values that added to video frames [14,15]. Images obtained from these surfaces by coherent imaging systems such as laser, SAR, and ultrasound suffer from a common phenomenon called speckle. Speckle, in both cases, is primarily due to the interference of the returning wave at the transducer aperture. The origin of this noise is seen if we model our reflectivity function as an array of scatterers. Because of the finite resolution, at any time we are receiving from a distribution of scatterers within the resolution cell. These scattered signals add coherently; that is, they add constructively and destructively depending on the relative phases of each scattered waveform. Speckle noise results from these patterns of constructive and destructive interference shown as bright and dark dots in the image [16].

Filtering techniques aim to reduce video degradation in order to increase efficacy of vehicle detection and tracking. The possible presence of salt \& pepper noise, Gaussian noise, and speckle video degradation could be reduced by using spatial filtering techniques. Methodology works robustly by applying the product of the filter mask with the neighbourhood pixels of each frame $[17,18]$. In this paper, some sort of spatial filtering techniques has been applied in order to satisfy the least deviation in the average number of assigned tracks $[19,20]$. The suggested filters were utilized as follow; average filter (Low Pass Filter) which depends on averaging the appropriate neighbourhood pixels in each frame, maximum, minimum, and median filters which depend on applying the appropriate mask according to ascending order based pixels, wiener filter: estimates the local mean and variance around each pixel as follow:

$$
\begin{gathered}
\mu=\frac{1}{M N} \sum_{n, m}^{N, M} p(n, m) \\
\sigma^{2}=\frac{1}{M N} \sum_{n, m}^{N, M} p(n, m)^{2}-\mu^{2} \\
\text { wiener filter estimator }= \\
\mu+\frac{\sigma^{2}-v^{2}}{\sigma^{2}}[p(n, m)-\mu]
\end{gathered}
$$

Where $v^{2}$ is the noise variance disk filter which affect neighborhood pixels according to circulated mask with a predefined radius of interest, laplacian and laplacian of Gaussian filters which use symmetrical mask based 
orthogonal matrix [21,22]. Mathematical representation of laplacian of Gaussian filter follow the equation:

$$
\operatorname{LOG}(x, y)=-\frac{1}{\pi \sigma^{4}}\left[1-\frac{x^{2}+y^{2}}{2 \sigma^{2}}\right] \exp \left(-\frac{x^{2}+y^{2}}{2 \sigma^{2}}\right)
$$

However, the Gaussian mathematical model follows the equation:

$$
G(x, y)=\frac{1}{2 \pi \sigma^{2}} \exp \left(-\frac{x^{2}+y^{2}}{2 \sigma^{2}}\right)
$$

In addition, motion, sobel, and prewitt filter mask have been applied with symmetrical based orthogonal masks which used for edge detection purposes [23]. Finally, Gaussian filter which used as a smoothing operator in order to blur frames and remove detail and noise. In this sense it is similar to the mean filter, but it uses a different kernel that represents the shape of a Gaussian bell-shaped hump. Vehicle detection and tracking would be analyzed according to all preciously discussed methodologies $[24,25]$.

\section{EXPERIMENTAL RESULTS}

The proposed study has been utilized and tested using Matlab 2015, 4 GB RAM, CORE I5 processor. A 500 frames traffic test video have been used for such analysis. Results have been divided into three levels; level1 expresses the process of estimating average number of assigned tracks in the raw traffic video (without any noise). By applying both foreground detection and Kalman filter algorithms, the average number of assigned tracks have been measured. The appropriate analysis of interest has recorded an averaging within 1.99 (average of all correct predicted vehicles in the 500 frames). Figure 2 shows all correct predicted vehicles in each frame. Horizontal axes represent video frames and vertical axes represents assigned tracks. Level2 of the proposed analysis expresses the degraded traffic video (by adding noise disturbances). In case of the presence of salt and pepper noise, gradually increasing based noise variance have been tested. Each time, the average number of assigned tracks have been recorded. It was obviously that there is a deviation in its value each time. Figure 3 shows the appropriate deviation according to the presence of salt and pepper noise. In case of the presence of both Gaussian and speckle noise, an obvious deviation has been witnesses in the tracking value. by increasing variance of the appropriate noise in both cases, deviation has recorded to be increased randomly. Figure 4 and Figure 5 show the effect of both Gaussian and speckle noise respectively. The last level of analysis aims to apply a bank of filter masks to the noisy traffic video in order to calculate the least deviation between reference value (no noise assigned track value) and deviated value (after cleaning assigned track value).

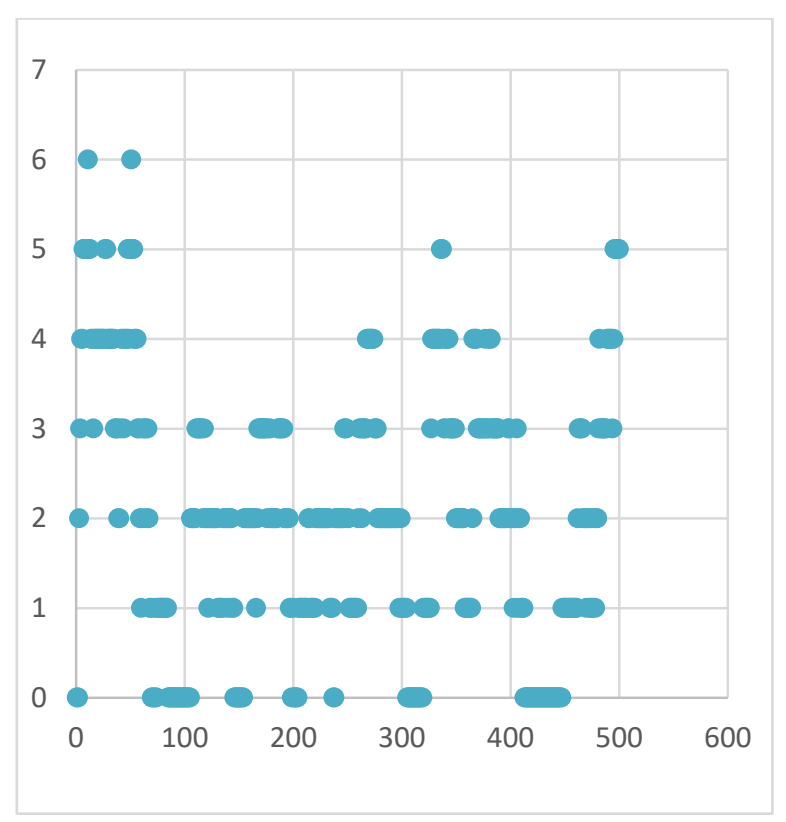

Fig.2. All assigned tracks in the whole test video

Figures 6, 7, and 8 show the effect of each filter in the tracking performance in the presence of each type of noise with different variance. Experimental results show that median filter has recorded the best performed filter with all types and cases. Median filter has targeting deviation values of 0.6 after cleaning salt and speckle noise, about 0.45 after cleaning Gaussian noise, and about 0.28 after cleaning salt and pepper noise.

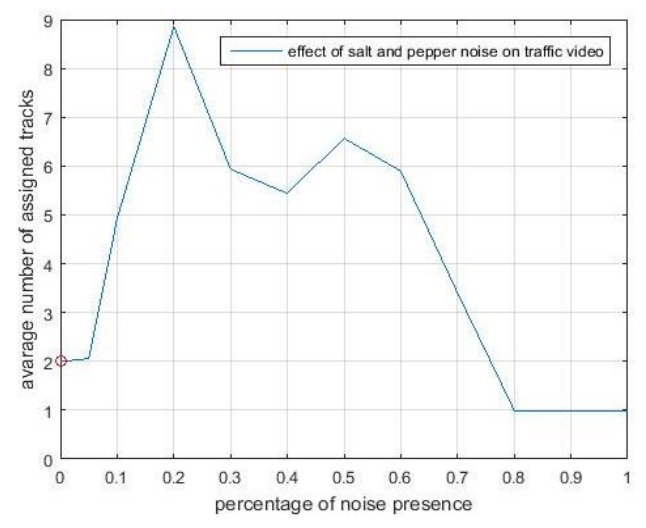

Fig.3. Effect of salt and pepper noise

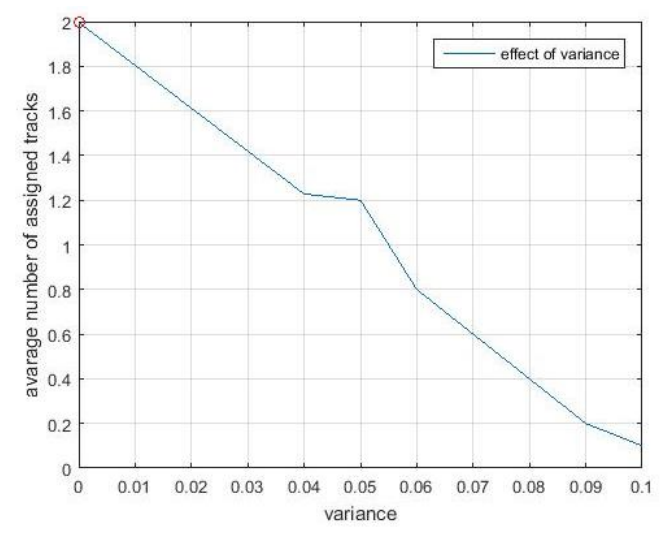

Fig.4. Effect of Gaussian noise 


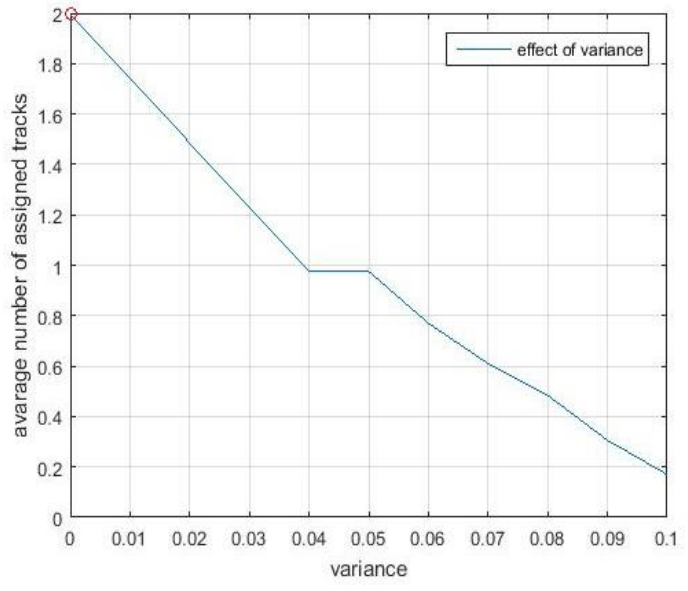

Fig.5. Effect of speckle noise

\section{CONCLUSION}

A complete traffic video analysis has been utilized and tested in the state of the art of computer vision based system. This work aims to ease the decision making of filtering system in case of dealing with noisy traffic videos. Performance indices in the appropriate analysis was targeting the measurement of the deviation between average number of assigned tracks in the noiseless traffic video and cleaned version from that video. We recommend the use of median filter in such cleaning criteria. Future work of interest will be in the state of the art of experiencing more filters with benchmarked traffic videos.

\section{REFERENCES}

[1] S.Sivaraman, M.M.Trivedi,"looking at vehicles on the road:A survey of vision based vehicle detection, tracking, and behavior analysis"IEEE transaction on intelligent transportation systems, VOL.14,NO.4, 2013.

[2] S. Sato, M. Hashimoto, M. Takita, K. Takagi, and T. Ogawa, "Multilayer lidar-based pedestrian tracking in urban environments," in Proc. IEEE IV, Jun. 2010, pp. 849-854.

[3] Z. Kim, "Real time obstacle detection and tracking based on constrained Delaunay triangulation," in Proc. IEEE ITSC, Sep. 2006, pp. 548-553. [30] J. Nuevo, I. Parra, J. Sjoberg, and L. Bergasa, "Estimating surrounding vehicles' pose using computer vision," in Proc. 13th ITSC, Sep. 2010, pp. 1863-1868.

[4] C. Idler, R. Schweiger, D. Paulus, M. Mahlisch, and W. Ritter, "Real time vision based multi-target-tracking with particle filters in automotive applications," in Proc. IEEE Intell. Veh. Symp., 2006, pp. 188-193.

[5] J. Nuevo, I. Parra, J. Sjoberg, and L. Bergasa, "Estimating surrounding vehicles' pose using computer vision," in Proc. 13th ITSC, Sep. 2010, pp. 1863-1868.

[6] M. K. Krishna, H. Hexmoor, and S. Sogani, "A t-step ahead constrained optimal target detection algorithm for a multi sensor surveillance system," IEEE Intelligent Robots and Systems, pp. 357-362 2005.

[7] W.Jing, B.Wen-xing. "A video supported moving object detection technique based on difference algorithm".
Computer Applications and Software, vol.26, no.12, pp: 68-70, 2009.

[8] Sun Ling, Wang Shuo, Li Qing-xia. "A target detection method based on adaptive threshold mixed difference". Computer Applications and Software, vol. 26, no. 10, pp: 94-97, 2009.

[9] Xiaopeng Chen, Qiang Huang, et al. "Rapid and Precise Object Detection based on Color Histograms and Adaptive Bandwidth Mean Shift". International Conference on Intelligent Robots and Systems, IROS 2009. IEEE/RSJ, pp: 4281-4286, 2009.

[10] R.Cucchiara, M.Piccardi, A.Prati, "Detecting Moving Objects, Ghosts, and Shadows in Video Streams", IEEE transactions on pattern analysis and machine intelligence, , october 2003 , vol. 25, no.10.

[11] O. Barnich and M. Van Droogenbroeck. Vibe,"A universal background subtraction algorithm for video sequences", IEEE Trans. Image Process., 20(6):1709-1724, June 2011

[12] J.-M. Guo, C.-H. Hsia, Y.-F. Liu, M.-H. Shih, C.-H. Chang, and J.-Y. Wu. "Fast background subtraction based on a multilayer codebook model for moving object detection", IEEE Trans. Circuits Syst. Video Technol., 23(10):1809-1821, Oct. 2013

[13] T. Bouwmans. "Traditional and recent approaches in background modeling for foreground detection", An overview. Computer Science Review, 1112(0):31-66,2014.

[14] L.Xue , C.Jiang, H.Chang , Y.Yang,W.Qin , W.Yuan , A novel Kalman filter for combining outputs of MEMS gyroscope array , elsevier, 4 January 2012 ,page(s)[745 746].

[15] J.Guo , B.M.Williams , W.Huang , Adaptive Kalman filter approach for stochastic short-term traffic flow rate prediction and uncertainty q uantification, Tranportation Research Part C: Emerging Technologies, Volume 43, Part 1 , June 2014 , page(s):50 \& 51

[16] L.Xue , C.Jiang, H.Chang , Y.Yang,W.Qin , W.Yuan , A novel Kalman filter for combining outputs of MEMS gyroscope array, elsevier, 4 January 2012 ,page(s)[745 746].

[17] C N .Ko , C M .Lee , Short-term load forecasting using SVR (support vector regression)-based radial basis function neural network with dual extended Kalman filter , elsevier, 14 December 2012, page(s)[413].

[18] M. Alam, J. Ferreira, J. Fonseca, "Introduction to Intelligent Transportation Systems", jpournal of intelligent transportation system. Studies in Systems, Decision and Control, vol 52. Pp.1-17, 2016. Springer

[19] X. Wang; R. Jiang; L. Li; Y. Lin; X. Zheng; F. Y. Wang, "Capturing Car-Following Behaviors by Deep Learning," in IEEE Transactions on Intelligent Transportation Systems, vol.99, 2017, pp.1-11

[20] J. K. Suhr; H. G. Jung, "Rearview Camera-Based Backover Warning System Exploiting a Combination of PoseSpecific Pedestrian Recognitions," in IEEE Transactions on Intelligent Transportation Systems, vol.99, 2017, pp.1 7.

[21] S. Kim, B. Coifman," Assessing the performance of Speed-Info radar traffic sensors", Journal of Intelligent Transportation Systems.pp.179-189, vol.21, 2017.

[22] M. Cobo, A. López-Herrera, F. Herrera, and E. HerreraViedma, "A note on the ITS topic evolution in the period 2000-2009 at T-ITS," IEEE Trans. Intelligent Transportation System, vol. 13, no. 1, pp. 413-420, Mar. 2012.

[23] D. Poobathy, R. Manicka Chezian,"Edge Detection Operators: Peak Signal to Noise Ratio Based Comparison", IJIGSP, vol.6, no.10, pp.55-61, 2014.DOI: 10.5815/ijigsp.2014. 
[24] N. Kulkarni," Color thresholding method for image segmentation of natural images", IJIGSP, vol.4, issue.1, pages 28,2012 . Comparative analysis of automatic vehicle
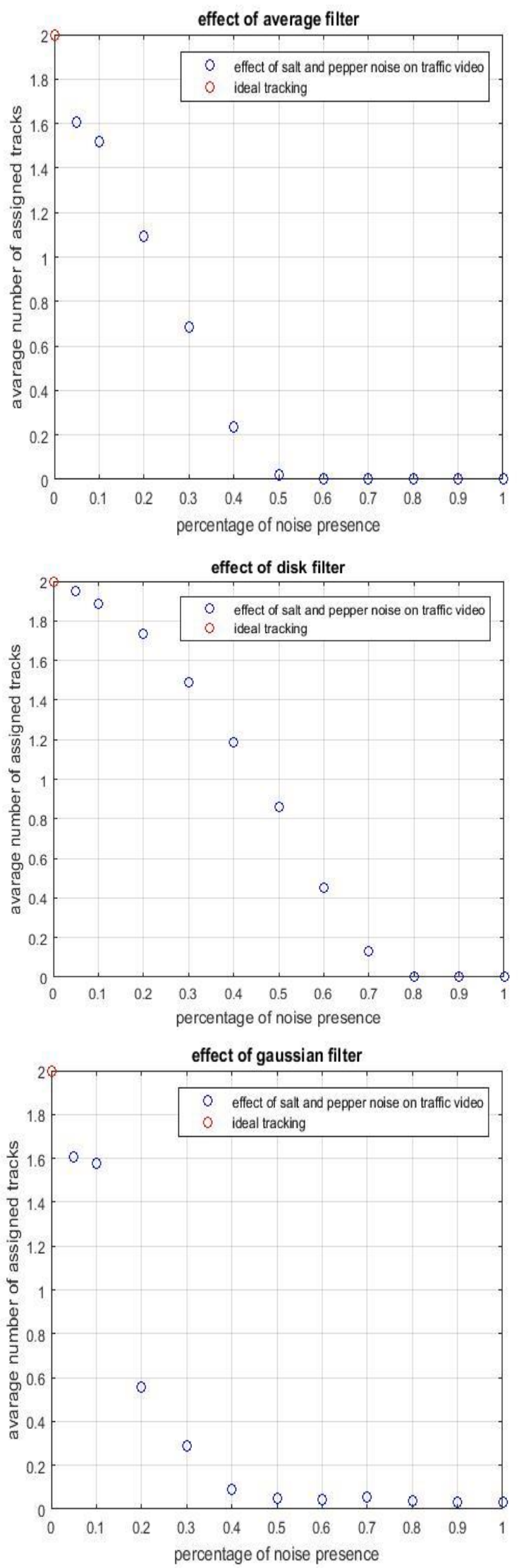

classification techniques: a survey", IJIGSP, vol.4, issue.9, pages 52, 2012.
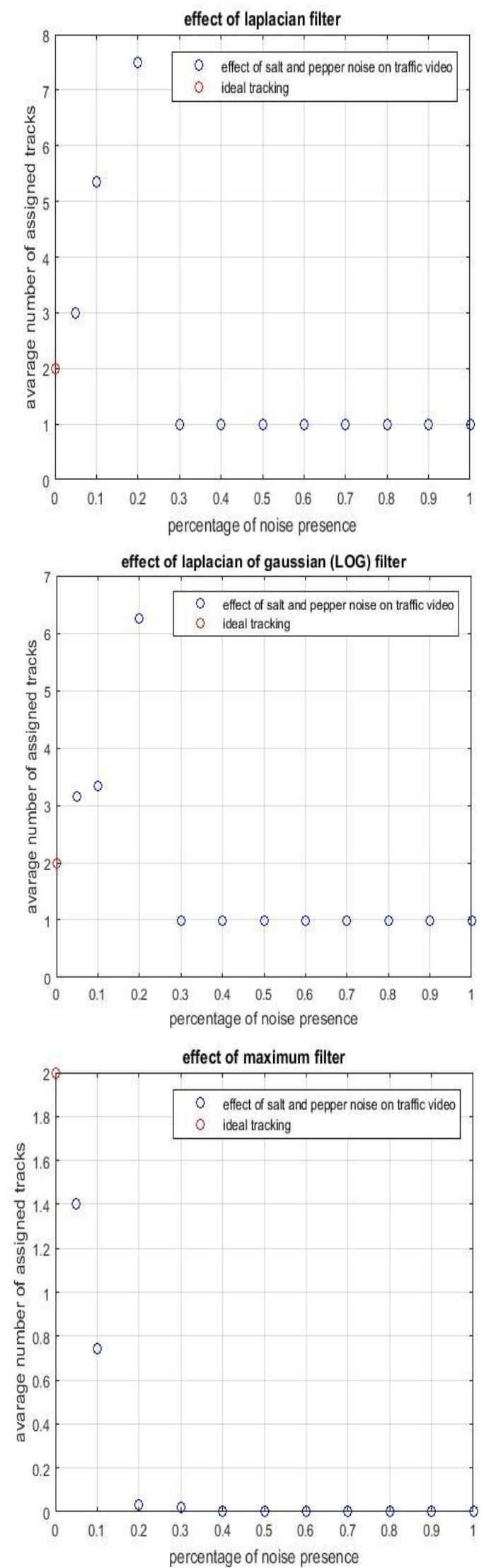

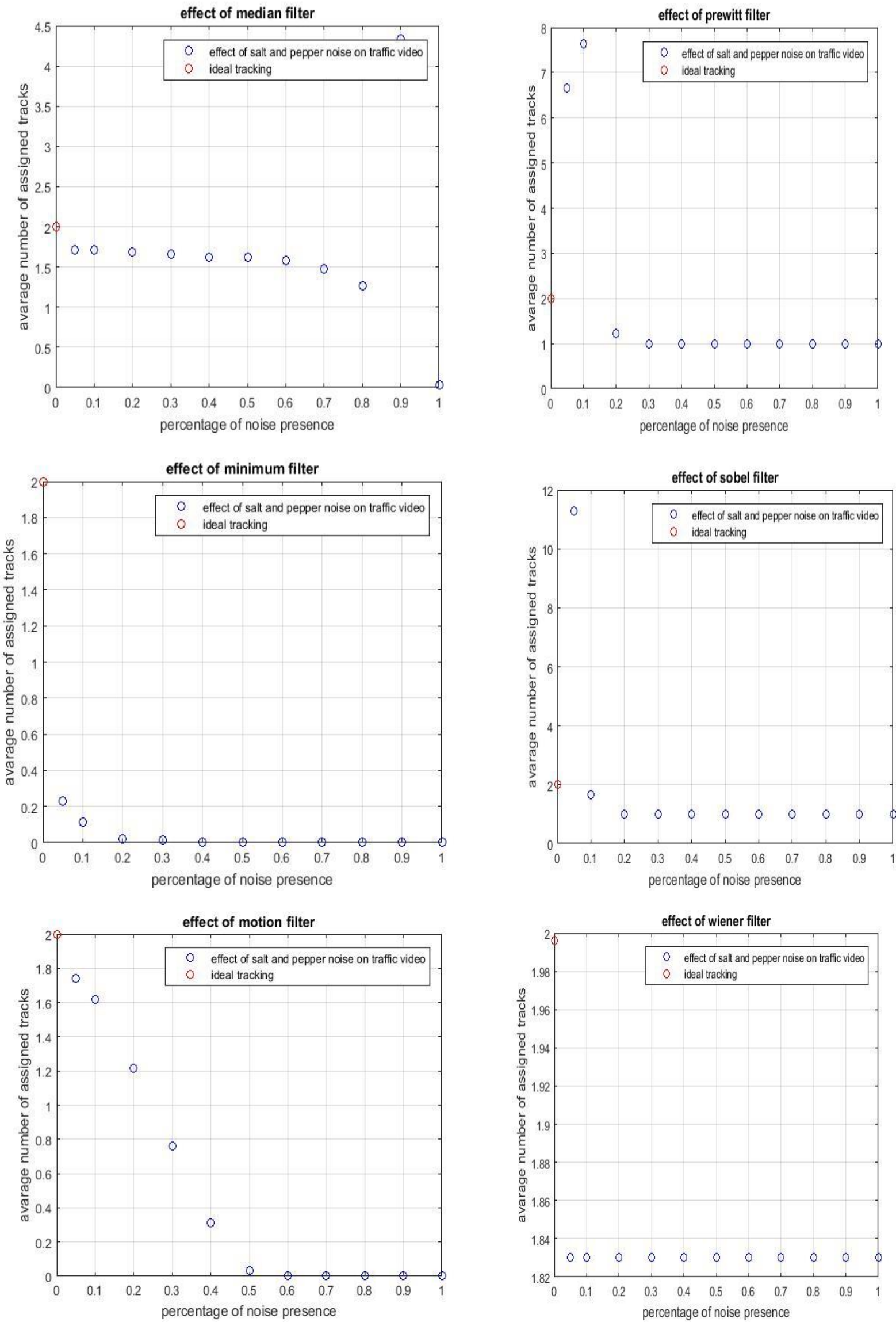

Fig.6. Deviation in value of assigned tracks after cleaning salt and pepper noise with all filter cases 

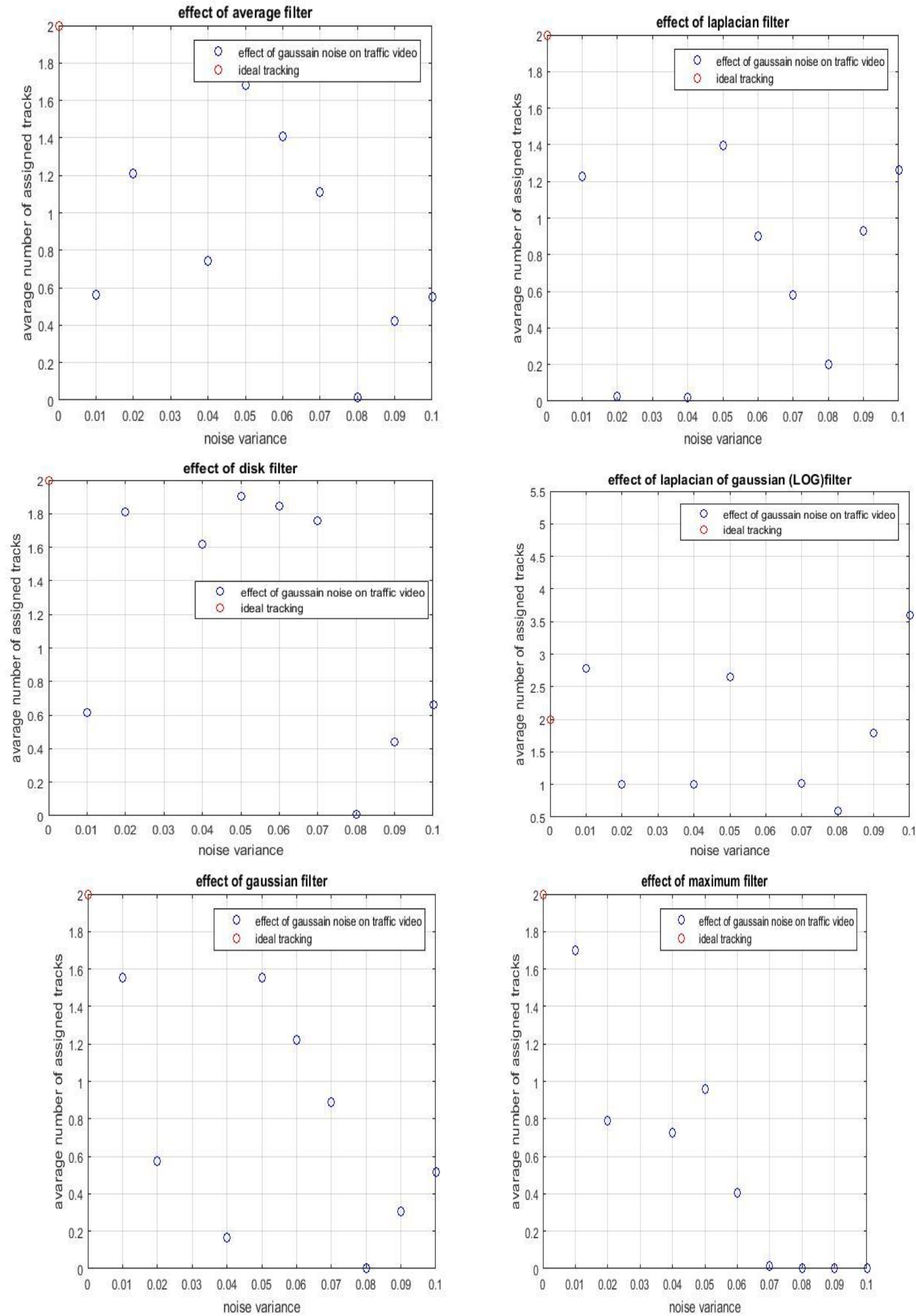

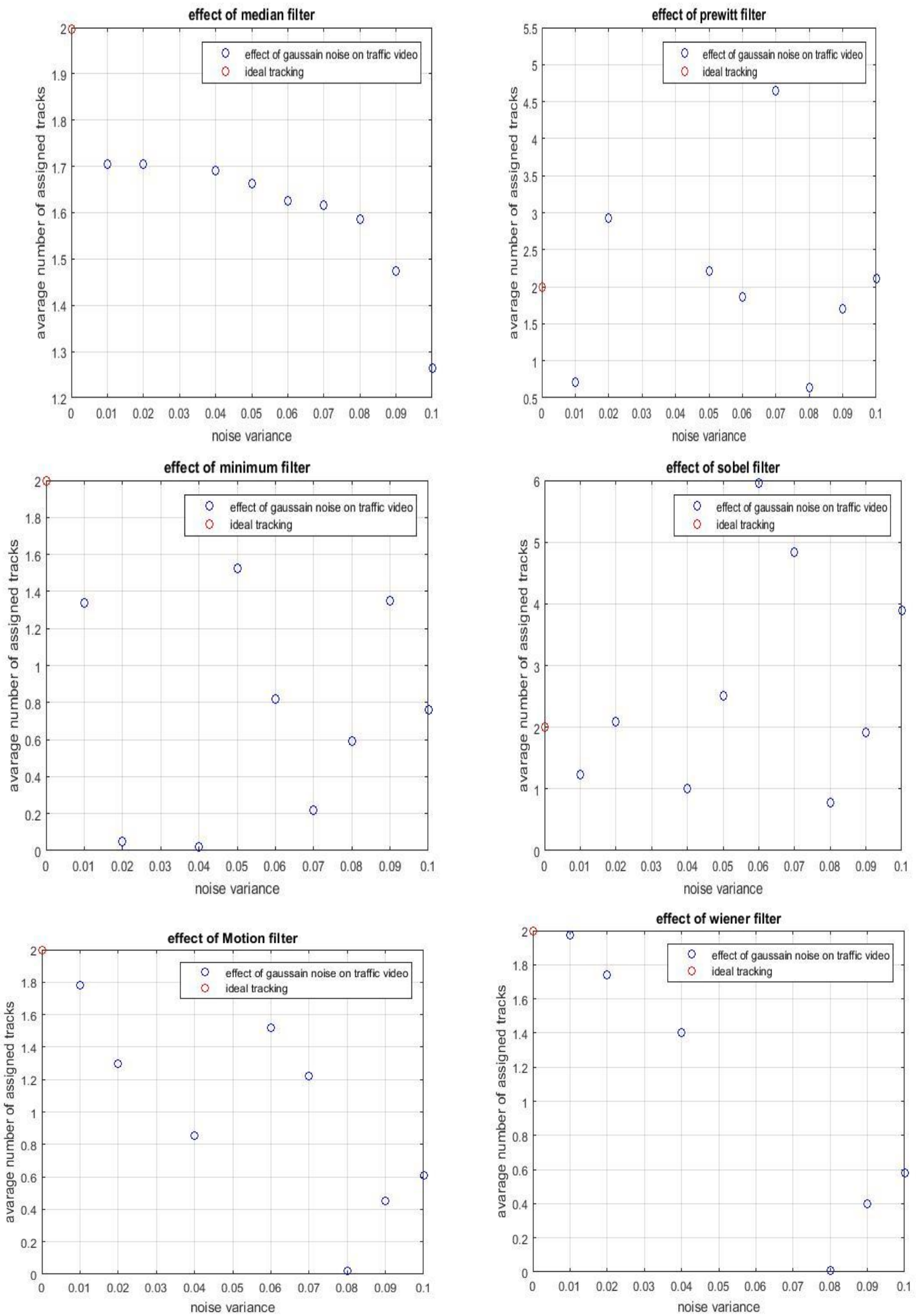

Fig.7. Deviation in value of assigned tracks after cleaning Gaussian noise with all filter cases 

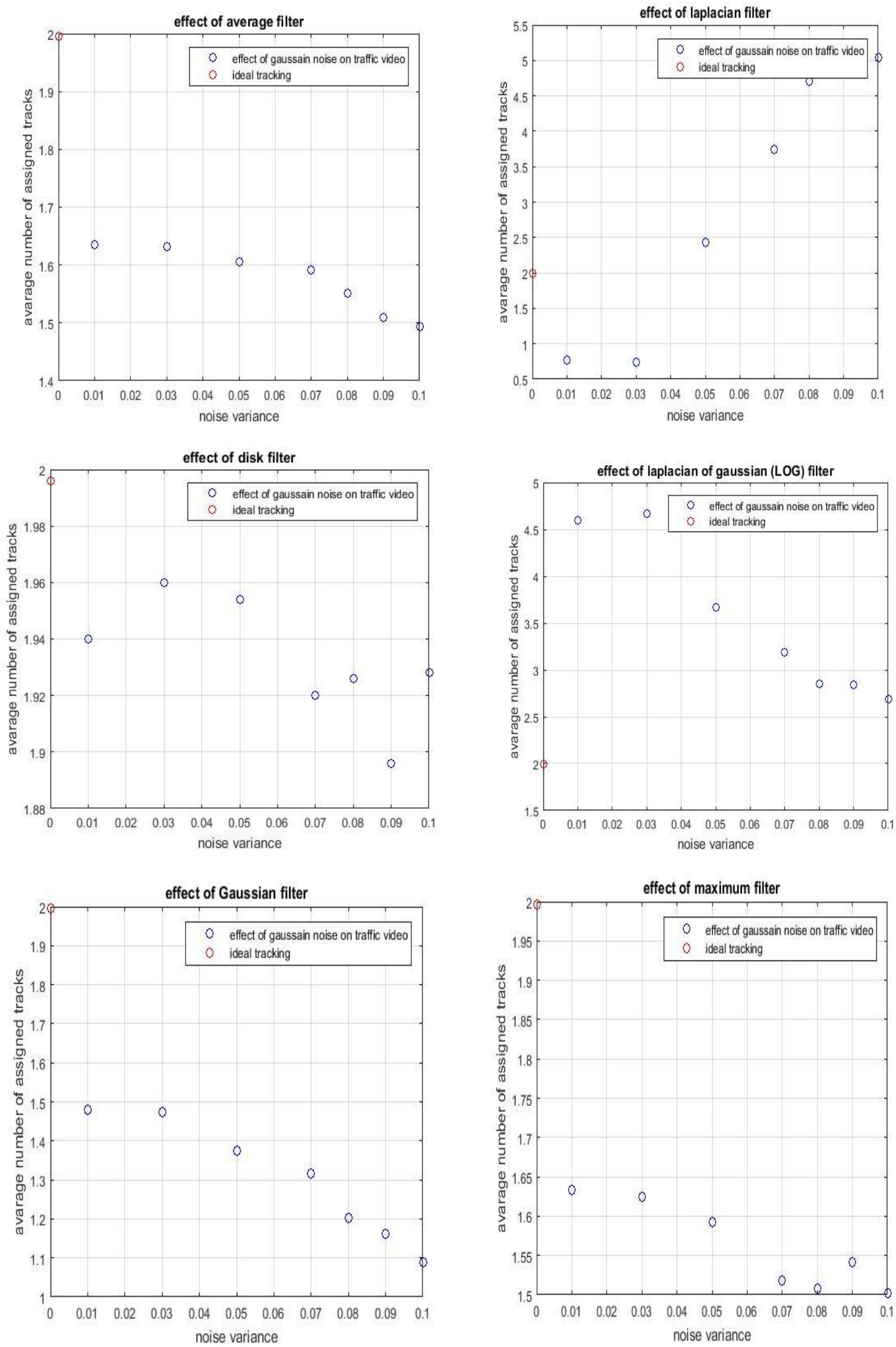

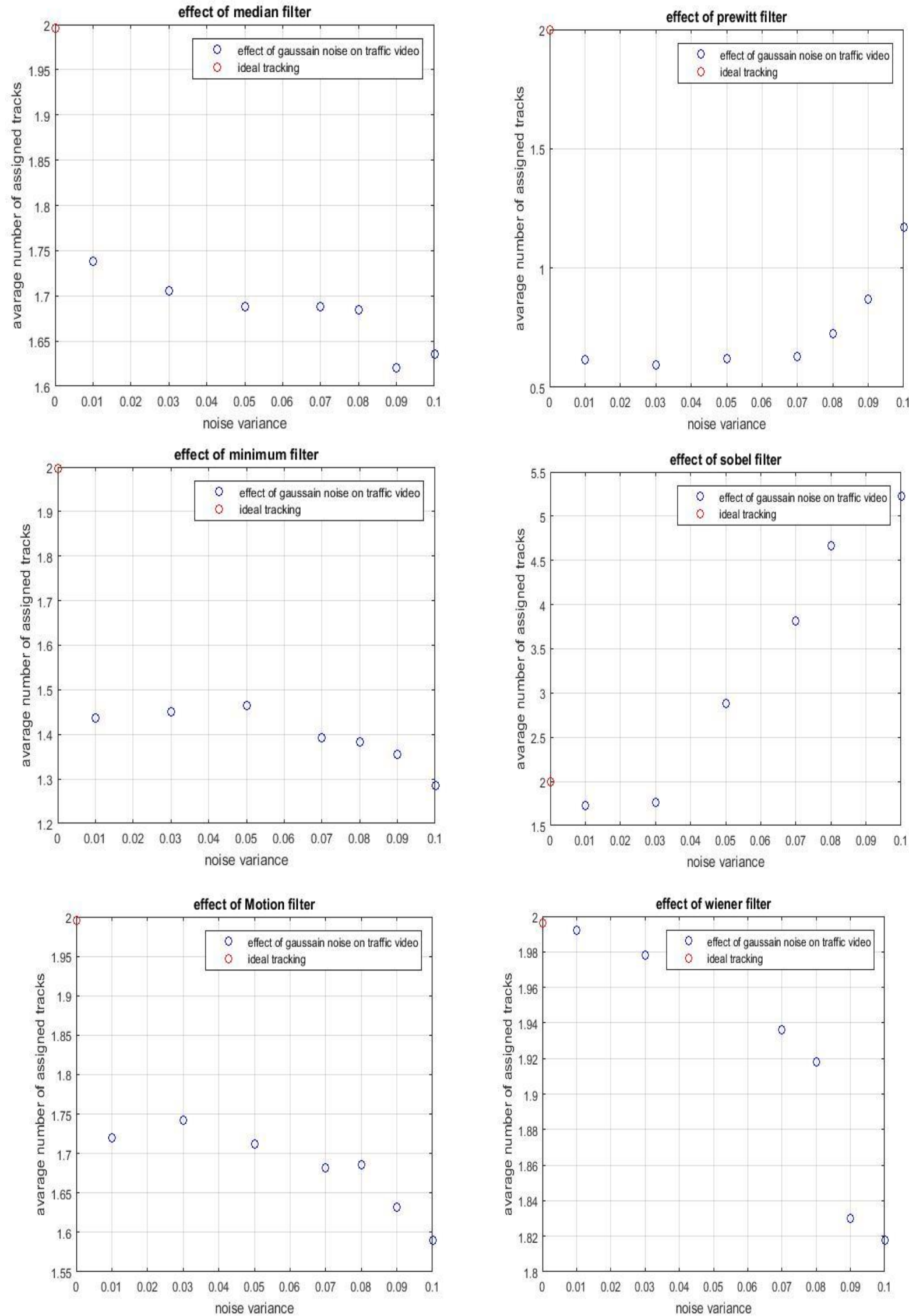

Fig.8. Deviation in value of assigned tracks after cleaning speckle noise with all filter cases 


\section{Authors' Profiles}

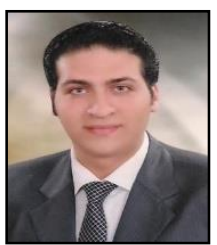

Mohamed Maher Ata is a $\mathrm{PhD}$ student, faculty of Engineering, Tanta university, Egypt. His research area of interest was utilized in the field of signal processing, image processing, Multimedia, and video processing. He has published many indexed research articles (SJR indexed-ISI indexedIET indexed) biomedical Engineering, astrophysics and intelligent transportation systems (ITS) which could be considered as a backbone of his $\mathrm{PhD}$ thesis of interest. He works as an assistant lecturer and teaches an advanced Matlab programming for Engineering applications, Matlab Simulink for Engineering applications, advanced mathematics, and undergraduate engineering courses. He is a member in science $\&$ Research support society (SERSC)

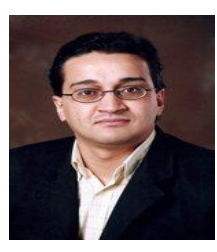

Mohamed El-Darieby (M'98) is an associate professor of Software Systems Engineering at the Faculty of Engineering at the University of Regina, Regina, Canada. $\mathrm{He}$ received B.Sc. and M.Sc.in electrical engineering from Egypt and Ph.D in Systems and Computer Engineering at Carleton University, Ottawa, Canada. His research is in the areas of backbone networks, grid computing and wireless mesh networks. Dr. El-Darieby is a member of the IEEE and the Association of Professional Engineers and Geoscientists of Saskatchewan (APEGS).

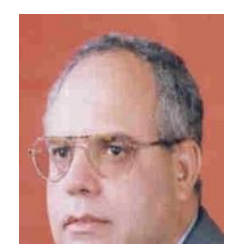

Mustafa Abdelnabi is a Professor of Electronics and Electrical Communication Engineering, Faculty of Engineering, Tanta University, Algarbia, Egypt. Field of specialization: Image processing, Medical imaging, Machine learning, Biomedical. Systems, Pattern recognition, Signal/image/video processing, Image analysis, Computer vision, Mathematical Modeling and Computer Simulation, Modeling of Biomedical Systems, Robotics and Systems, Computer Aided Diagnosis and Optimization. Total number of publications in Scopus about 88 publications

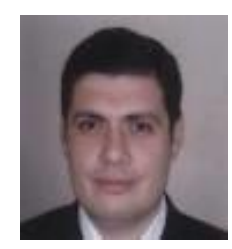

Sameh A. Napoleon got his B.Sc. in the field of Electronics and Electrical Communication Engineering from the Faculty of Engineering, Tanta University, Egypt in 1999. He received the M.Sc. and the Ph.D. from Tanta University in 2006 and 2012 respectively. The objectives of the M.Sc. thesis were to test performance of different types of voice coders over ATM and IP networks and to optimize the QoS for voice traffic via optimizing and developing forward packet loss recovery techniques. His Ph.D. introduces an efficient and applicable replacement to GPS for positioning in indoor environments by using the WLAN access points to find the accurate position of any mobile device in that special multipath environment. His research interests are localization and tracking techniques and sensor networks.

How to cite this paper: Mohamed Maher Ata, Mohamed El-Darieby, M.Abd Elnaby, Sameh A. Napoleon," Traffic Video Enhancement based Vehicle Correct Tracked Methodology", International Journal of Image, Graphics and Signal Processing(IJIGSP), Vol.9, No.12, pp. 30-40, 2017.DOI: 10.5815/ijigsp.2017.12.04 